\title{
The effects of laser illumination and high energy electrons on molecular-beam epitaxial growth of CdTe
}

\author{
Y. S. Wu, ${ }^{\text {a) }}$ C. R. Becker, A. Waag, R. N. Bicknell-Tassius, and G. Landwehr \\ Physikalisches Institut der Universität Würzburg, D-8700 Würzburg, Germany
}

(Received 25 May 1990; accepted for publication 21 August 1990)

\begin{abstract}
We report the results of a detailed investigation on the Te-stabilized $(2 \times 1)$ and the Cdstabilized $c(2 \times 2)$ surfaces of $(100)$ CdTe substrates. The investigation demonstrates for the first time that both laser illumination and, to a greater extent, high-energy electron irradiation increase the Te desorption and reduce the Cd desorption from (100) CdTe surfaces. Thus it is possible by choosing the proper growth temperature and photon or electron fluxes to change the surface reconstruction from the normally Te-stabilized to a Cd-stabilized phase.
\end{abstract}

\section{INTRODUCTION}

The use of light to improve the quality of molecularbeam epitaxially (MBE) grown II-VI materials and to enhance substitutional doping is one of the recent exciting developments in the growth of compound semiconductors. Bicknell et al. and Hwang et al. ${ }^{1-3}$ have grown high-quality $\mathrm{CdTe}$ with this photoassisted molecular-beam epitaxy (PAMBE) method. Their best undoped $n$-type CdTe had a mobility of $6600 \mathrm{~cm}^{2} / \mathrm{Vs}$ and their In-doped CdTe $\left(n=2 \times 10^{16} \mathrm{~cm}^{-3}\right.$ at $\left.300 \mathrm{~K}\right)$ had a mobility of 2380 $\mathrm{cm}^{2} / \mathrm{V} \mathrm{s}$. This is in contrast to conventional MBE-grown CdTe which is semi-insulating. ${ }^{4}$ Due to the availability of high-quality conducting epilayers of $\mathrm{CdTe}$, all thin-film $\mathrm{CdTe}$ electronic devices have been recently produced, i.e., $p$ $n$ junctions and metal-semiconductor field-effect transistors. ${ }^{5,6}$ Photoassisted molecular-beam epitaxy has also been applied to the growth of highly conducting, dilute magnetic semiconductor epitaxial layers and superlattices. ${ }^{7}$ Recently, this new technique has been applied to the growth of mercury-based II-VI materials. Koestner et al. have grown (100) and (211) $\mathrm{HgCdTe}$ epitaxial layers using PAMBE which exhibited a low dislocation density, $1-2 \times 10^{5}$ and 3$5 \times 10^{4} \mathrm{~cm}^{-2}$, respectively, over a wider range of growth conditions than is possible by conventional MBE. ${ }^{8}$ Similarly Myers et al. have grown some of the best epitaxial $\mathrm{HgCdTe}$ to date with extremely narrow $\mathrm{x}$-ray double-crystal rocking curves and very high carrier mobility. ${ }^{9}$ They reported dislocation line densities for (211) $\mathrm{HgCdTe}$ as low as $5 \times 10^{4}$ $\mathrm{cm}^{-2}$. Their reported electron mobilities of the (100) $\mathrm{HgCdTe}$ epilayer are as high as $8.5 \times 10^{5} \mathrm{~cm}^{2} / \mathrm{V}$ s for samples with $13 \% \mathrm{Cd}$, and are $4 \times 10^{5} \mathrm{~cm}^{2} / \mathrm{V}$ s for a sample with $20 \% \mathrm{Cd}$.

The above results may be summarized in general by stating that photoillumination during $\mathrm{MBE}$ growth greatly improves the structural properties as well as the electrical properties of the epitaxial layers that are grown using the photoassisted MBE technique. Therefore, detailed investigations into the mechanisms of how light affects the growing surface is of utmost importance. If the mechanism of the photoassisted MBE process is understood in detail it should

\footnotetext{
a) Permanent address: Institute of Physics, Chinese Academy of Sciences, Beijing, People's Republic of China.
}

be easier to optimize the technique for any one material and easier to apply it to other materials systems. In fact, it has been recently reported that UV light illumination can enhance the migration of adsorbates of dissociated adatoms on $\mathrm{GaAs}$ surfaces and thereby improve the GaAs quality grown by molecular layer epitaxy. ${ }^{10,11}$

To date, only a limited number of investigations have been carried out that give insight into the microscopic mechanisms of the PAMBE process. Benson et al. have shown that laser illumination enhances Te desorption and produces more sites for $\mathrm{Sb}$ incorporation. ${ }^{12}$ It has also been shown that the use of an excess cadmium flux during the MBE growth of CdTe has a similar effect to that obtained by the PAMBE technique. ${ }^{13,14}$ An attempt to explain the effects of illumination on compound semiconductor surfaces during epitaxial growth has been made by Farrell et al. who have proposed a model to describe the mechanisms by which light interacts with surface species during thin-film growth using MBE or other related techniques. ${ }^{15}$

Normally the region within the MBE system that is directly involved in the growth process can be divided into three distinct parts: the crystalline phase, the gaseous phase, and the interface or the transition layer between these two. The crystalline epitaxial layer exhibits long-range order in the spatial distribution of the constituent atoms. In contrast, the gaseous phase near the substrate surface consisting of molecular and atomic beams is characterized by a complete lack of long-range spatial order. The interface between these two phases where all processes leading to epitaxy occur is obviously the most important part of the growth system. Its geometrical form and the processes occurring there depend strongly on the growth conditions chosen. Therefore, it is critical for the growth of ideal films to recognize and to properly adjust the structure and compositions of this interface layer.

To our knowledge no publication has reported on laserinduced effects on the CdTe growing surface except for the studies of the static CdTe surface by Benson et al. ${ }^{12,16}$ In addition, only a few studies of the effect of high-energy electrons (HEE) on the surface chemistry have appeared in the literature, even though one of the most widely used in situ surface analysis techniques is reflection high-energy electron diffraction (RHEED). In fact, irradiation of the CdTe surface during growth by high-energy electrons strongly in- 
fluences the surface stoichiometry and therefore the observed surface reconstruction. Thus, this brings into question the applicability of RHEED and other such techniques to the investigation of CdTe surfaces. In this paper we present observations of the effects of laser illumination and HEE irradiation on the growing surface of CdTe epitaxial layers.

The experiments that are reported in this paper should be contrasted with the recent investigations of the effects of post-growth electron irradiation of CdTe surfaces that showed that such irradiation produces deep level defects in the sample. ${ }^{17}$ In the present experiments we have illuminated the CdTe at a glancing incidence angle $\left(\approx 1^{\circ}\right)$, which is typical of those used in RHEED studies. At such low incidence angles the penetration depth of the electrons into the sample surface is limited to the first few atomic layers. Due to this very shallow penetration the effects of the electron illumination is limited to the growth interface.

\section{EXPERIMENTAL DETAILS}

Epitaxial growth was carried out in a four-chamber RIBER 2300 MBE system. The MBE system has been modified to permit illumination of the substrate during the film growth process. Two MBE sources were employed in the surface phase diagram studies. One contained high-purity polycrystalline CdTe and the other contained high-purity cadmium. It should be noted that while the $\mathrm{Cd}$ source was of conventional "open-tube" design, as is available from several commerical suppliers, the CdTe source was of Knudsen cell design. The orifice of the CdTe cell was $3 \mathrm{~mm}$ in diameter, and should help to ensure that near-equilibrium conditions exist within the effusion cell. In the present work, typical operating temperatures of the $\mathrm{CdTe}$ and $\mathrm{Cd}$ sources were 750 and $150^{\circ} \mathrm{C}$, respectively. The vacuum in the $\mathrm{CdTe}$ growth chamber was better than $6 \times 10^{-10}$ Torr. In order to ensure that $\mathrm{Cd}$ or $\mathrm{CdTe}$ fluxes escaping around the effusion cell shutters did not effect the experimental results, a second shutter close to the substrate was also used. The substrate temperature was measured with an accuracy of $\pm 2{ }^{\circ} \mathrm{C}$ by means of a thermocouple which was in actual physical contact with the molybdenum substrate holder. The thermocouple was carefully calibrated at the melting points of indium and tin.

The substrates were (100) CdTe wafers which were chemomechanically polished for several minutes, degreased using standard solvents, etched in a weak bromine/methanol solution, and rinsed in methanol. Immediately prior to loading the substrates into the MBE system, they were rinsed in de-ionized water, briefly dipped in hydrochloric acid, and then rinsed in de-ionized water so as to remove any remaining oxide and carbon on the substrate surface.

The CdTe substrates were preheated at $100^{\circ} \mathrm{C}$ for 15 min and then the temperature was slowly raised up to 300$400{ }^{\circ} \mathrm{C}$ depending on the substrate surface which was monitored with reflection high-energy electron diffraction (RHEED). The use of in situ x-ray photoelectron spectroscopy (XPS) helped to ensure that only optimally cleaned surfaces were used in the following investigations. ${ }^{18,19}$

A RIBER model CER 606 electron gun with model
ACE 1010 controlling electronics was used as a source of high-energy electrons both for the RHEED patterns and in the investigations of the effects of high-energy electrons on the epitaxial growth surfaces. In the present experiments, the acceleration voltage of the electron gun was about $9 \mathrm{kV}$, the electron current was about $20 \mu \mathrm{A}$, and the beam was focused as small as possible. A Coherent Inova 90-6 Argonion laser $(514.5 \mathrm{~nm})$ was used to produce the photons that were employed in the PAMBE experiments. This laser allowed the entire growth surface to be uniformly illuminated with power densities up to $500 \mathrm{~mW} / \mathrm{cm}^{2}$. The power densities incident on the surface were determined using a Coherent 210 power meter. In the present work power densities of up to $320 \mathrm{~mW} / \mathrm{cm}^{2}$ were employed. At these power densities the rise in the substrate surface temperature is expected to be smaller than $1^{\circ} \mathrm{C} .^{4,20}$

\section{RESULTS AND DISCUSSION}

In general, the Te-stabilized surface is smooth and its RHEED pattern in the [011] azimuth displays half-order reconstruction (HOR). ${ }^{21}$ The Cd-stabilized surface is rougher and the corresponding RHEED patterns in the [010] and [001] azimuths also display HOR. There is a mixed region in which HOR appears in the [011], [010], and [001] azimuths. In particular, HOR in the [011] azimuth always appears even though the CdTe surface is under a Cd-rich environment, i.e., excess Cd flux. Therefore, in this work we have used the appearance of a very weak HOR in the [010] azimuth as a limit for determining the desorption time of excess Te on the surface. In other words, the first appearance of excess $\mathrm{Cd}$ on the surface denotes the desorption of excess $\mathrm{Te}$.

\section{A. Static CdTe surfaces}

Figure 1 shows the effect of laser illumination and HEE on the "static" CdTe surface, i.e., without cadmium, tellurium, or CdTe fluxes impinging on the surface. In these experiments the CdTe flux $\left(3 \times 10^{-7}\right.$ Torr) was stopped after

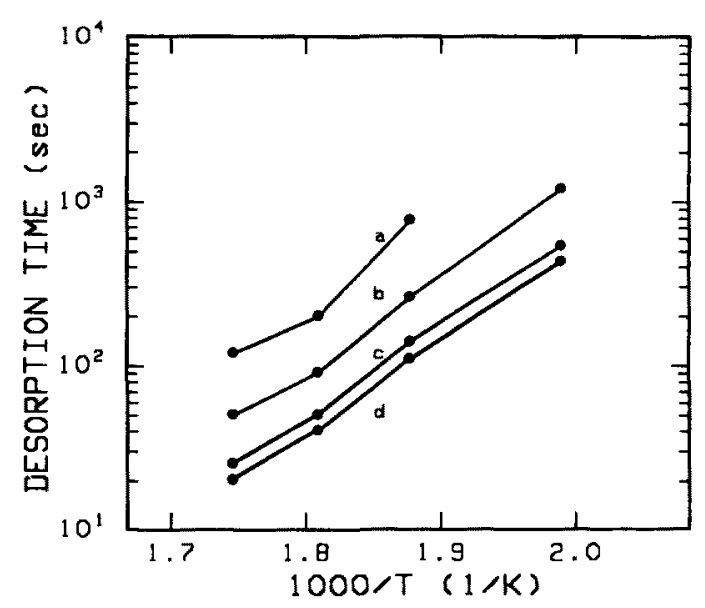

FIG. 1. Desorption time of excess Te on the static surface with (a) neither laser nor high-energy electron (HEE) irradiation, (b) laser illumination, (c) HEE irradiation, and (d) both the laser illumination and the HEE irradiation. The laser intensity was $45 \pm 5 \mathrm{~mW} / \mathrm{cm}^{2}$. 
growing a thin CdTe layer by conventional MBE for $15 \mathrm{~min}$ prior to every measurement. Under these conditions the CdTe surfaces show HOR in the [011] azimuth. After closing the shutter to stop the CdTe growth the transition layer changes gradually with time. Curve (a) in Fig. 1 displays the desorption time of excess $\mathrm{Te}$ atoms on the surface as determined by the substrate temperature at which HOR in the [010] azimuth appeared. The substrate surface was not irradiated by HEE or photons. The time spent on checking RHEED patterns was as short as possible to minimize the effects of illumination by high-energy electrons. Normally about 2-3 $s$ were needed to observe the RHEED pattern. This was achieved using a switch to change the $x-y$ deviation voltages of the electron gun.

At $230^{\circ} \mathrm{C}$ no HOR in the [010] azimuth could be found even after 100 min. Curves (b), (c), and (d) were obtained under laser illumination, HEE irradiation, and both, respectively. It is obvious from Fig. 1 that both laser illumination and HEE irradiation of the substrate accelerated the desorption of Te, whereby the effect of the HEE irradiation was appreciably larger. The fact that laser-illumination induces tellurium desorption from $\mathrm{CdTe}$ surfaces is consistent with the experimental results of Benson et al. ${ }^{12}$

\section{B. Dynamic CdTe growth surfaces}

Laser illumination and HEE irradiation also affect the $\mathrm{CdTe}$ surface during growth. Table I lists the substrate temperature ranges over which half-order reconstruction in the [010] azimuth occurs for various growth conditions. The CdTe flux was $1.67 \times 10^{-7}$ Torr and the incident laser intensity was $200 \mathrm{~mW} / \mathrm{cm}^{2}$. Corresponding to this CdTe flux, the growth rate at $320^{\circ} \mathrm{C}$ was $0.4 \AA / \mathrm{s}$, which was measured in situ using RHEED oscillations. The appearance of the HOR in the [010] azimuth was determined with the naked eye. No evidence for HOR in the [010] azimuth was found outside the indicated temperature ranging during growth with the indicated growth conditions (i.e., laser illumination, HEE irradiation, etc). As shown in Table I, HOR in the [010] azimuth could be observed only in a very narrow temperature range between 350 and $356^{\circ} \mathrm{C}$ when conventional MBE growth techniques were employed. This means that outside this small temperature range the surface is tellurium stabilized. This is due to a combination of several factors: the $\mathrm{Cd}$ to-Te flux ratio of our CdTe Knudsen source, the $\mathrm{Cd}$ and $\mathrm{Te}$ sticking coefficients, and the reevaporation rates from the surface.

TABLE I. Temperature ranges over which half-orders reconstruction along the [010] azimuth occur under various growth conditions.

\begin{tabular}{lcc}
\hline \hline & \multicolumn{2}{c}{ Temperature range $\left({ }^{\circ} \mathrm{C}\right)$} \\
\cline { 2 - 3 } Conditions & Lower temperature & Upper temperature \\
\hline Neither laser nor high- & 350 & 356 \\
$\quad$ energy electrons & 338 & 364 \\
Laser & 316 & 374 \\
High-energy electrons & 310 & 386 \\
Laser and high-energy & & \\
electrons &
\end{tabular}

Irradiating the CdTe growing surface with photons, HEE or both greatly increased the temperature range over which HOR in the [010] azimuth appeared, as indicated in Table I. Therefore, the conclusion can be drawn that laser illumination and HEE irradiation increase the Cd content on the surface and extend the temperature range over which HOR in the [010] azimuth can be observed.

The effect of laser illumination on the CdTe growing surface is dependent on the incident laser intensity, as shown in Fig. 2. In the following experiment the CdTe flux was $5 \times 10^{-8}$ Torr, which corresponds to a growth rate of approximately 2 monolayers per minute. At the beginning we grew CdTe at $345^{\circ} \mathrm{C}$ on the prepared (100) CdTe substrate and slowly lowered the substrate temperature until HOR in the [010] azimuth disappeared. Then we increased the laser power until HOR in the [010] azimuth appeared. In this way we found that HOR in the [010] azimuth cannot be found if the substrate temperature was less than about $340^{\circ} \mathrm{C}$ and if a laser intensity less than $45 \mathrm{~mW} / \mathrm{cm}^{2}$ was employed.

The other points in Fig. 2 with the exception of points $A$ and $B$ indicate that HOR in the [010] azimuth appeared at the indicated substrate temperature when a laser intensity laser than the value indicated by the point was employed.

To summarize, HOR in the [010] azimuth, which is indicative of a Cd-stabilized surface, was observed in region II and was not observed in region I. In contrast, HOR in the [011] azimuth was visible at all temperatures and laser intensities investigated.

From the curves in Fig. 2 we can make the following statements: Firstly, there is a laser power threshold, i.e., HOR in the [010] azimuth cannot be found if the illumination power density is less than a limiting value. Secondly, laser intensities ranging from 50 to $160 \mathrm{~mW} / \mathrm{cm}^{2}$ can induce large changes in the substrate temperature region over which HOR in the [010] azimuth can be observed. Lastly, below a certain temperature HOR in the [010] azimuth cannot be observed independent of the illumination intensity employed. These observations are consistent with the work

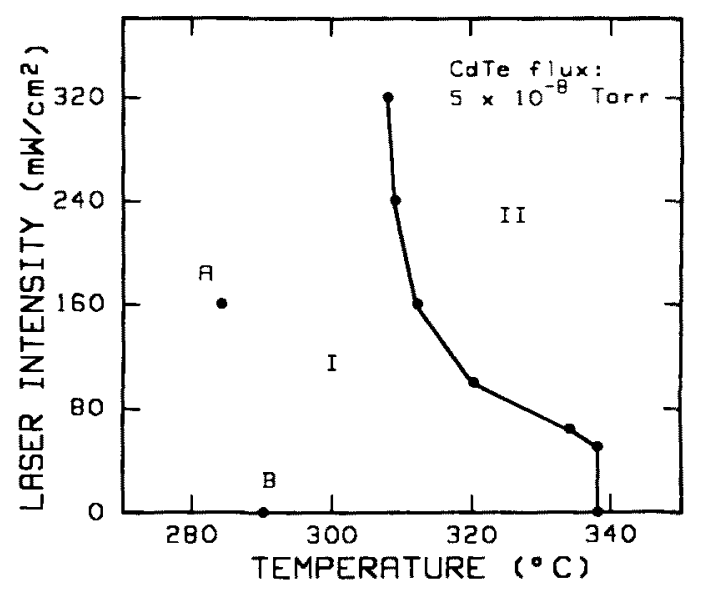

FIG. 2. Laser intensity vs substrate temperatures. Region I: no HOR in the [010] azimuth appeared. Region II: HOR in the [010] azimuth appeared. Points $\mathbf{A}$ and $\mathbf{B}$ were obtained in HEE irradiation and corresponding laser illumination 
of Bicknell-Tassius et al., which indicated that to optimize the photoluminescence spectra from CdTe epilayers grown by photoassisted MBE there was a resonance in the power density employed during the PAMBE process. ${ }^{22}$

In the above experiments the effects of illumination by high-energy electrons was also noted. If during these experiments the RHEED pattern was observed for more than about $10 \mathrm{~s}$, HOR in the [010] azimuth was much more clearly apparent unless the substrate temperature was smaller than the temperatures defined by points $\mathrm{A}$ and $\mathrm{B}$ corresponding to growth with and without laser illumination, respectively. This fact clearly demonstrates again the large effect HEE irradiation has on the surface. Here the growth rate was very low, about 2 monolayers per minute, and the surface was optically smooth. We propose that the MBE system was nearly in equilibrium. At this low growth rate we could observe and maintain these phenomena over a longer period of time.

Laser illumination and HEE irradiation has also been observed to affect the desorption of $\mathrm{Cd}$ from Cd-stabilized surfaces. In these experiments a CdTe epilayer was grown at $300{ }^{\circ} \mathrm{C}$ on the prepared (100) CdTe substrate for several minutes, using conventional MBE, photoassisted MBE, or HEE irradiation during the MBE growth process. The results described below were independent of the growth procedure employed. In this way a smooth Te-stabilized surface showing a $(2 \times 1)$ reconstruction was obtained. The CdTe source shutter was closed and the $\mathrm{Cd}$ source shutter was opened for $1 \mathrm{~min}$. The $\mathrm{CdTe}$ and $\mathrm{Cd}$ source fluxes employed were $3 \times 10^{-7}$ and $7 \times 10^{-6}$ Torr, respectively. After closing the $\mathrm{Cd}$ source shutter the surface exhibited a $c(2 \times 2)$ reconstruction, typical of a Cd-stabilized surface.

To determine the lifetime of excess $\mathrm{Cd}$ on this surface the elapsed time for the appearance of HOR in the [03 $\overline{1}]$ azimuth, which is $18.4^{\circ} \pm 1^{\circ}$ displaced from the [010] azimuth, was measured. The reconstruction along the [03i] azimuth rather than along [010] azimuth was used to determine the Cd surface lifetime. The reason for this is that the Te-stabilized $(2 \times 1)$ reconstructed surface surface displays strong HOR in the [011] azimuth and very clear but weaker HOR in the $[03 \overline{1}]$ azimuth. The Cd-stabilized $c(2 \times 2)$ reconstructed surface surface shows a very strong HOR in the [010] azimuth, a weak HOR in the [011] azimuth, and no HOR in the $[03 \overline{1}]$ azimuth. The HOR in the $[03 \overline{1}]$ azimuth appeared when the intensity of the HOR in the [011] and [010] azimuths were almost identical. Obviously this indicates that the surface is now neither Te nor Cd stabilized, but is at some intermediate or mixed stage. The process for the surface to change from the Cd-stabilized $c(2 \times 2)$ phase to the mixed phase is due to the desorption of excess $\mathrm{Cd}$. Therefore, we have chosen the appearance of HOR in the [03i] ] azimuth as a measure for the desorption time of excess $\mathrm{Cd}$ on the Cd-stabilized surface.

In this way we found the persistence time of the excess $\mathrm{Cd}$ on the CdTe surface at $300^{\circ} \mathrm{C}$ was 118,26 , and $16 \mathrm{~min}$ with HEE irradiation, laser illumination, and neither one, respectively. Therefore, it is apparent that laser illumination and high-energy electron illumination of the surface decreases the Cd desorption rate. The surface lifetime of excess
$\mathrm{Cd}$ on the surface under HEE irradiation at $300^{\circ} \mathrm{C}$ is consistent with the data presented in Ref. 16. Again it is apparent that the effects observed upon illuminating the surface with high-energy electrons is very similar to what is observed when the surface is illuminated with photons.

\section{CONCLUSIONS}

The results of this investigation can be summarized as follows. Firstly, the high-energy photons of an Ar-ion laser increases the desorption of Te from (100) CdTe surfaces. The opposite is true of Cd, i.e., the desorption is decreased upon illumination with photons. Secondly, both of these above statements are true when the (100) CdTe epitaxial layer is irradiated with HEE, but to a much larger extent. Lastly, there is a laser intensity range over which a $c(2 \times 2)$ Cd-stabilized surface can be observed. It is possible that this is related to the optimum laser intensity for the growth of high-quality CdTe epitaxial layers.

The effects observed under high-energy electron illumination are new and we believed very exciting. Effects very similar to those obtained with photon illumination have been obtained. Whether highly conducting CdTe epilayers can be obtained using HEE irradiation is not known, but if it is possible this effect could have many new and interesting applications. Such an effect would allow the control of the electrical properties in the growth plane by selective illumination of the epilayer surface with the high-energy electrons. This would be much easier with HEE illumination than with photons since the steering of the electron beam is quite trivial. Further studies are under way to investigate the electrical properties of the HEE illuminated epilayers.

From a more short-term perspective the results presented in this paper should lead anyone using RHEED to characterize growth surfaces to take a careful look to be sure that they are not changing what they want to look at by irradiating their surfaces with high energy electrons.

\section{ACKNOWLEDGMENTS}

This project was supported by the Bundeministerium für Forschung und Technologie and the Deutsche Forschungsgemeinschaft (Bonn).

'R. N. Bicknell, N. C. Giles, and J. F. Schetzina, Appl. Phys. Lett. 49, 1095 (1986).

${ }^{2}$ R. N. Bicknell, N. C. Giles, and J. F. Schetzina, Appl. Phys. Lett. 49, 1735 (1986).

${ }^{3}$ S. Hwang, R. L. Harper, K. A. Harris, N. C. Giles, R. N. Bicknell, R. M. Kolbas, and J. F. Schetzina, J. Vac. Sci. Technol. B 6, 777 (1988).

${ }^{4}$ R. N. Bicknell, N. C. Giles, and J. F. Schetzina, J. Vac. Sci. Technol. A 5, 3059 (1987).

${ }^{5}$ D. L. Dreifus, R. M. Kolbas, K. A. Harris, R. N. Bicknell, R. L. Harper, and J. F. Schetzina, Appl. Phys. Lett. 51, 931 ( 1987).

${ }^{6}$ D. Dreifus, R. Kolbas, J. Tassintino, R. Harper, R. Bicknell and J. Schetzina, J. Vac. Sci. Technol. A 6, 2722 (1988)

${ }^{7}$ R. L. Harper, R. N. Bicknell, D. K. Blanks, N. C. Giles, J. Schetzina, Y. R. Lee, and A. K. Ramdas, J. Appl. Phys. 65, 624 (1989).

${ }^{8}$ R. J. Koestner, H.-Y. Liu, H. Schaake, and T. Hanlon, J. Vac. Sci. Technol. A 7, 517 (1989).

${ }^{9}$ T. Myers, R. Yanka, K. Harris, A. Reisinger, J. Han, S. Hwang, Z. Yang, N. Giles, J. J. W. Cook, J. Schetzina, R. Green, and S. McDivitt, J. Vac. Sci. Technol. 7, 300 (1989). 
${ }^{10}$ J. Nishizawa, H. Abe, T. Kurabayshi, and N. Sakurai, J. Vac. Sci. Technol. A 4, 706 (1986).

"J. Nishizawa, T. Kurabayshi, and J. Hoshina, J. Electrochem. Soc. 134, 502 (1987).

'2J. Benson, D. Rajavel, B. Wagner, R. Benz II, and C. J. Summers, J. Cryst. Growth 95, 543 (1989).

${ }^{13}$ R. N. Bicknell-Tassius, A. Waag, Y. S. Wu, T. A. Kuhn, and W. Ossau, J. Cryst. Growth (in press).

${ }^{14}$ Y. S. Wu, A. Waag, and R. Bicknell-Tassius, (unpublished).

${ }^{15}$ H. Farrell, X. Nahory, and J. Harbison, J. Vac. Sci. Technol. B 6, 779 (1988).

${ }^{16} \mathrm{~J}$. Benson. B. Wagner, A. Torabi, and C. J. Summers, Appl. Phys. Lett. 49,
1034 (1986).

${ }^{17}$ J. Shaw, L. Brillson, S. Sivanathan, and J. Faurie, Appl. Phys. Lett. 56, 1266 (1990).

${ }^{14}$ A. Waag, Y. S. Wu, R. N. Bicknell-Tassius, and G. Landwehr, Appl. Phys. Lett. 54, 2662 (1989)

${ }^{19}$ A. Waag, Y. S. Wu, R. N. Bicknell-Tassius, C. Gonser-Buntrock, and G. Lanwehr, J. Appl. Phys. 68, 212 (1990).

${ }^{20} \mathrm{C}$. Uzan, R. Legros, Y. Marfaing, and R. Triboulet, Appl. Phys. Lett. 45, 879 (1984).

${ }^{21}$ J. Singh and J. Arias, J. Vac. Sci. Technol. A 7, 2562 (1989).

${ }^{22}$ R. N. Bicknell-Tassius, T. A. Kuhn, and W. Ossau, Appl. Surf. Sci. 36, 95 (1989). 